\title{
Laparoscopic Boari flap formation in advanced ovarian carcinoma
}

\author{
Niamh Daly, ${ }^{1}$ Alison DeMaio, ${ }^{1}$ Ronan Daly, ${ }^{1}$ Feras Abu Saadeh ${ }^{1,2}$
}

${ }^{1}$ Department of Gynaecological Oncology, St. James's Hospital, Dublin 8, Ireland

${ }^{2}$ Trinity College Department of Obstetrics and Gynaecology, Trinity Centre for Health Sciences, St. James's Hospital, Dublin 8, Ireland

\section{Correspondence to}

Dr Feras Abu Saadeh, Department of Gynaecological Oncology, St. James's Hospital, Dublin 8, Ireland; ferasabusaadeh@dubgyn.org

Accepted 20 April 2020 Published Online First 28 May 2020
Check for updates

C IGCS and ESGO 2020. No commercial re-use. See rights and permissions. Published by BMJ.

To cite: Daly N, DeMaio A, Daly R, et al. Int J Gynecol Cancer 2020;30:1081.

\section{OBJECTIVE}

To demonstrate laparoscopic ureteric reconstruction with formation of a Boari flap.

\section{METHODS}

We present video footage of a laparoscopic Boari flap formation in a patient with previous extensive primary debulking surgery and stenosis of a prior excision of the distal ureter with a Boari flap and stabilizing psoas hitch formation. ${ }^{1}$ The woman underwent adjuvant chemotherapy for stage IIIC high grade serous ovarian cancer and presented subsequently with ureteric stenosis.

\section{RESULTS}

The animation in the video outlines the key steps of this laparoscopic procedure, including identification of the ureter at the bifurcation of the common iliac artery, and ureterolysis to mobilize the ureter caudally from the pelvic side wall and the external iliac vein. We guide the surgeon as the bladder is placed on traction anteriorly and avascular peritoneal planes are identified and dissected, and the bladder mobilized bilaterally. The bladder is then filled to optimize cold-cutting (to avoid thermal injury) of a U-shaped incision of the anterior bladder to outline the Boari flap. We illustrate transection and spatulation of the ureter before re-implantation into the mucosa of the Boari flap, with demonstration of the interrupted intracorporeal suturing technique. A double $\mathrm{J}$ stent (24/7 French) is cystoscopically inserted through the bladder into the ureter over a guide wire. We show tubularisation of the flap and closure of the bladder with interrupted intracorporeal suturing techniques.

The indwelling urinary catheter was removed at 6 weeks and the ureteric stent was removed at 12 weeks post-procedure. A follow-up cystogram demonstrated bladder integrity. The woman is now 1 year postprocedure and remains asymptomatic with normal renal function biochemistry and ultrasound.

Laparoscopic Boari flap formation is described in the literature with excellent success rates. ${ }^{23}$ To our knowledge this is the first report in a patient with

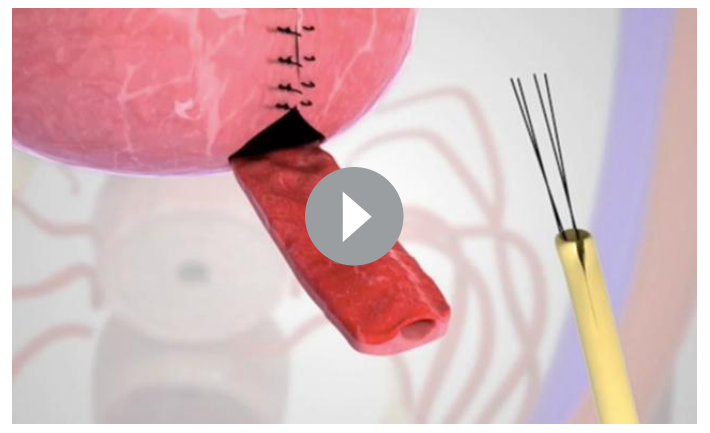

Video 1 Still images of laparoscopic.

stenosis of a prior Boari flap formation during initial debulking surgery that was followed by adjuvant chemotherapy for advanced ovarian cancer.

\section{Conclusions}

Through the use of animation, video and still images we present laparoscopic ureteric reconstruction with the formation of a Boari flap.

Contributors Each author made a substantial contribution to the conception and design of the video and writing and editing of this original abstract. Each has contributed to drafting the video or revising it critically for important intellectual content, and has approved the final submitted video and abstract.

Funding The authors have not declared a specific grant for this research from any funding agency in the public, commercial or not-for-profit sectors.

Competing interests None declared.

Patient consent for publication Not required.

Provenance and peer review Not commissioned; externally peer reviewed.

Data availability statement There are no data in this work

\section{REFERENCES}

1 Aminsharifi A. Minimally invasive management of concomitant vesicovaginal and ureterovaginal fistulas after transabdominal hysterectomy: laparoscopic vesicovaginal fistula repair with ureteroneocystostomy using a Boari flap. J Minim Invasive Gynecol 2018;25:17-18.

2 Seideman CA, Huckabay C, Smith KD, et al. Laparoscopic ureteral reimplantation: technique and outcomes. J Urol 2009;181:1742-6.

3 Andrade C, Narducci F, Bresson L, et al. Boari flap ureteroneocystostomy in an oncological patient. Gynecol Oncol 2016;143:193. 